\title{
Identification information sensors of robot systems
}

\author{
Igor Parkhomey ${ }^{1}$, Juliy Boiko², Oleksander Eromenko ${ }^{3}$ \\ ${ }^{1}$ Technical Cybernetics Department, National Technical University of Ukraine, \\ Igor Sikorsky Kyiv Polytechnic Institute, Ukraine \\ ${ }^{2}$ Department of Telecommunications and Radio Engineering, Khmelnytsky National University, Ukraine \\ ${ }^{3}$ Department of Physics and Electrical Engineering, Khmelnytsky National University, Ukraine
}

\begin{tabular}{l}
\hline \hline Article Info \\
\hline Article history: \\
Received Dec 22, 2018 \\
Revised Jan 21, 2019 \\
Accepted Feb 28, 2019 \\
\hline
\end{tabular}

\section{Keywords:}

Autocorrelation function

Coefficient of similarity

Description of images

Image border

Invariance

\begin{abstract}
At the present time, the complexity of identification is to find such a description, in which the image (information) of each class would have identified similar properties. The task is to make the transformed description includes the whole set of input images, united by the similarity class by the given ratio. Using the ordinates of an autocorrelation function is an inseparable shift in the center of gravity of an image, which leads to a change of such description. Nicest, the concept of an invariant description of information arises, this is an autocorrelation function, which is invariant to the description of any displacements of the image in the vertical and horizontal directions. The problem of finding an optimal decision rule arises, which, in a number of cases, can be constructed on the basis of a method, based on the definition of the maximum incomplete coefficient of similarity. Using this method, the solutions, that are almost unintelligible to the errors that arise due to the effects of interference, are found. Therefore, in increments $\mathrm{k}$, this rule passes into the Bayes' rule.
\end{abstract}

Copyright () 2019 Institute of Advanced Engineering and Science. All rights reserved.

\section{Corresponding Author:}

Juliy Boiko,

Department of Telecommunications and Radio Engineering,

Khmelnytsky National University, Ukraine.

Email: boiko_julius@ukr.net

\section{INTRODUCTION}

In the works of authors such as [1]-[9] and other. It is noted that in practice for solving these problems, when the classification process is based on incomplete data, the problem of finding an optimal deciding rule arises, the methods of determination of which are based on the definition of the maximum incomplete coefficient of similarity.

However, these methods do not take into account the effect of interference on the recognition process. Because often in the training sequence images are presented that are not subject to interference, and in the process of recognition, analyzed images distorted by noise.

So, in order to eliminate the effects of interference on the process of recognition, it is proposed to introduce a special decree rule that is based on the fact that the decision on the affiliation of the image to the image is made on the basis of an analysis of images that have fallen into a certain closest space, which is classified.

\section{RESEARCH METHOD}

Choosing a description of images is one of the important tasks in pattern recognition. It is known, that the most complete description of the input images can be represented as a continuous function of two variables, which describes the distribution of brightness across the receptor field $Z(x, y)$. The completeness of 
these input images description has a number of significant disadvantages: the description is too cumbersome; it is not invariant with respect to many isomorphic transformations of the images.

The ability of generalizations of the recognition system's response to similar input images is required, regardless of their location in the retina field, on the internal interactions and interactions of the system. To ensure the ability of generalizations of the reaction, there are usually some impose limitations, which depend on the nature of the similarity ratio. The restrictions, imposed on the logical nature of the similarity ratio, are they should have the property of symmetry and reflectivity, that is, if $A$ is like $B$, then $B$ is like $A$, and $A$ is always like itself.

There are a large number of similarity ratios in nature, which these conditions are inherent in. These include: the solid motion of the image, some types of continuous deformations, topological equivalence of the images. Solid motion refers to the rotation and movement of the image, which is parallel to the horizontal or vertical axis.

\subsection{Recognition Systems Problems}

Recognition systems, possessing the property of generalization by similarity, are characterized by significant structural complications (for example, an increase in the number of layers of $A$-elements in perceptron) compared with conventional systems. Therefore, it is desirable to pre-convert the original description in such a way as to generalize all such objects and to react, in a differentiated way, to objects that are not similar [10], [11].

The class of similarity in a given ratio stands for the set of input images, similar in this ratio. It is believed, that the similarity ratio is the imposition of an image so that $A \cup B / R$, which means $A$ is similar to $B$ in relation $R$ (where $R$ is the relativity of the motion). This means that $A$ is a mapping of the displacement $B$, and $B$ is a reflection of the motion of $A$. The problem is that the transformed description includes the whole set of input images, united by the similarity class by the given ration. The autocorrelation function carries sufficiently complete information about the character of the input image [12]-[15] in addition, it is invariant towards to the description of moving images in the vertical and horizontal directions.

To ensure the invariance of the description to the movement of the image, it is enough to use the ordinates of the autocorrelation function as the description elements. However, the shift in the center of gravity of the image leads to a change in such a description. To reduce the effect of the shift of the center of gravity on the description of the input image, which is compiled from the ordinates of such an autocorrelation function, one should exclude from the description ordinates, corresponding to a rotation angle close to $0^{\circ}$ and $180^{\circ}$.

It is desirable to obtain such a description that would cover the whole set of input images, united by the similarity class of all displacements of images relative to the receptor field. Such a description is invariant with respect to any displacement of an image. In order to obtain an invariant description, with respect to all the rigid displacements, it is necessary to create a fundamentally new type of scan. If two random functions $x=x(t)$ and $y=y(t)$ are put on the camera's deflection system, then the beam will move randomly on the screen, and the signal from the camera will be a random function of the time, the statistical characteristics of which depend on both the nature of the input image and on the properties of the functions $x=x(t)$ and $y=y(t)$.

The multivariate distribution of the output signal gives it a complete probabilistic characteristic. It is obvious, that with stable probabilistic characteristics of functions $x=x(t)$ and $y=y(t)$ the probabilistic characteristics of the output signal will depend only on the nature of the input image. This statement is proved in [16]-[19].

If the function $Z=Z(x, y)$, where $x=x(t)$ and $y=y(t)$. Then if $x=x(t)$ and $y=y(t)$ are stationary and stationary-random functions of the time with even distribution and a vector, which consists of $x$ and $y$, characterized by a uniformly distribution of probability, then the function $Z(t)$ is stationary in the broad and narrow sense; and its probabilistic characteristics do not depend on the inverses transformations and parallel transfer of the function $Z(x, y)$.

It directly follows from this, that the ordinates of the autocorrelation function $A_{Z}\left(t, t^{\prime}\right)$ depend only on $\tau=t-t^{\prime}$ and the nature of the dependence $Z(x, y)$ and do not depend on the inverse transformations and the parallel transfer of the function.

Thus, the description of the input image, composed of the ordinates of the autocorrelation function of the output signal of the television camera, under certain restrictions imposed on the signal, is invariant with respect to all the rigid movements of the image inside the screen (the image is arbitrarily moved on the screen, without leaving any of its elements beyond its limits). 


\subsection{The Possible Methods for Describing the Image}

It would seem, that using a random scan and description of the image by the ordinates of the autocorrelation function, solves a very complicated problem of recognition. However, the practical implementation of such a system has significant technical difficulties. First, it is quite difficult to fulfill all the constraints imposed on the signals $x(t)$ and $y(t)$. In addition, the proved theorem relates only to the qualitative aspect of the question and does not relate to the quantitative one. The autocorrelation function $A_{Z \tau}$ does not really depend on the movement of the image on the screen, but, at the same time, its difference from the representation of the images is so small, that it is practically impossible to distinguish autocorrelation functions, even when the images, corresponding to these functions, are quite different from each other.

The autocorrelation function of the stationary signal is uniquely connected by Fourier transform with its spectral density:

$$
A_{Z}\left(t, t^{\prime}\right)=A_{Z}(\tau)=\int_{0}^{\infty} f_{Z}(\omega) \cos \omega \tau \mathrm{d} \omega
$$

where $\left.f_{\not z} \omega\right)$ - spectral density of the signal and vice versa

$$
f_{Z}(\omega)=\frac{2}{\pi} \int_{0}^{\infty} A_{Z}(\tau) \cos \omega \tau \mathrm{d} \tau
$$

Therefore, statements, concerning the autocorrelation function, are also valid for spectral density.

If the output signal of the camera $Z(t)$ goes to the analyzer, which consists of a finite number of filters with uniform frequency characteristics, the resonance frequencies of which are located one by one on the axis $\omega$. Let's suppose that the output signals of the filters are reduced to a square, and then they are integrated. Output signals of such analyzer are determined by the distribution of the energy $W_{Z}(\omega)$ of the signal $Z(t)$ along the frequency axis.

It is known, that the weighted autocorrelation function of a signal $A_{Z}(T) q(T)$ is related to the Fourier transform with its energy spectrum $W_{Z}(\omega)$ :

$$
g(\tau)=\int_{0}^{\infty} K^{2}(\omega) e^{-j \omega t}
$$

where $K(\omega)$ - frequency response of the filter.

Therefore, the description of the input image can be formed using the ordinate of the energy spectrum.

Thus, each of the functions $A_{Z}(T), f_{Z}(\omega)$ or $W_{Z}(\omega)$ may be convenient for describing the input images when recognizing images. However, it is much easier to get a characteristic of the spectral density of a signal than its autocorrelation function.

In [20-23] one of the possible methods for describing the image is considered with the help of the spectral characteristics of the signal obtained in the frame scan of the image. To simplify considerations, images with only two degrees of intensity of information are considered, but all this is true for images with a continuous change in intensity.

In the general case, the signal, received at the output of the television camera, carries all information about the scanned black and white image. As a rule, this signal can always be represented by an infinite Fourier series:

$$
f(x)=\frac{a_{0}}{2}+a_{1} \cos \omega x+b_{1} \sin \omega x+a_{2} \cos 2 \omega x+b_{2} \sin 2 \omega x+\cdots,
$$

Where,

$a_{k}=\frac{2}{T} \int_{0}^{T} f(x) \cos k \omega x d x, b_{k}=\frac{2}{T} \int_{0}^{T} f(x) \sin k \omega x d x$.

Since a television signal has a finite spectrum, then an infinite Fourier series can be written as finite sum:

$$
f_{n}(x)=\frac{a_{0}}{2}+\sum_{k=1}^{n} a_{k} \cos k \omega x+\sum_{k=1}^{n} b_{k} \sin k \omega x
$$


The function $f_{n}(x)$, and hence the image to which it corresponds, can be given by the coefficients of this series, which is equal to the representation of the image or coefficients of the Fourier series, or ordinates of spectral density.

The television signal that corresponds to two intensity gradations is a sequence of rectangular impulses of the same amplitude, all of which is contained in the duration of impulses and pauses. In addition, the television signal contains more string and frame impulses, their amplitude are $25 \%$ higher amplitude of useful impulses.

The recognition procedure is greatly complicated with linear and frame impulses, firstly, because they clog the useful signal, and secondly, because their presence leads to significant changes in the spectral density of the signal. This is due to the fact that the offsets and turns of the image lead to a change in the duration and recognition of the useful impulses relative to the string impulses is shown in Figure 1.

In order to eliminate the effect of the image shift, which is parallel to the axis of the receptor field, it is necessary to remove linear and frame impulses from the TV signal. In this case, the image shift affects only the phase of the signal and does not affect its spectrum.

To eliminate the effect of the rotation, you need to rotate the image on the receptive field of the vidicon and, as a feature, take the average ordinate of the spectrum in one rotation. In this way, we get the same results as when randomly scanned.

However, it may be that images, belonging to different classes, differ little from the spectral component. To distinguish such images, it is necessary to choose not the average values of ordinates per rotation, but the functions of their instantaneous values in time, that is, to take into account the redistribution of the spectral density of the signal during one rotation. The problem of recognition, in this case, is greatly complicated by the fact that the description of images throughout the volume increases sharply. At the same time, it cannot be guaranteed that the transformed description will be less than the original, but it acquires useful properties of invariance with respect to offsets and turns.

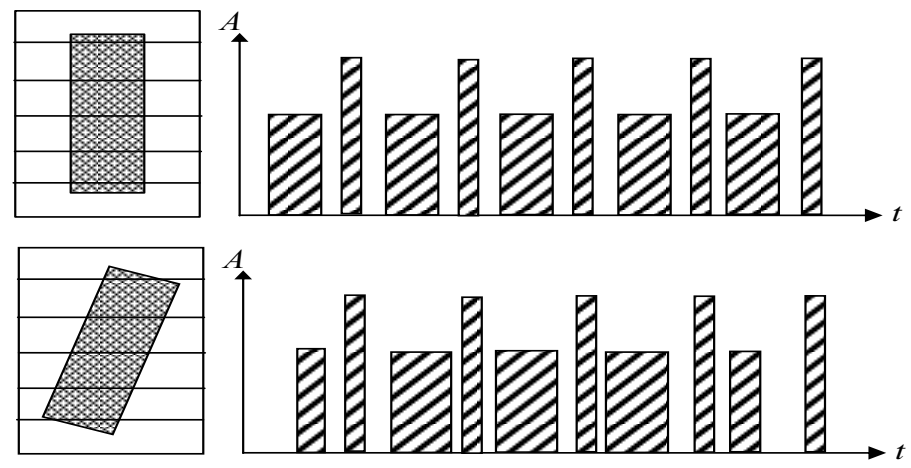

Figure 1. Scanning an image

Taking into account the periodic nature of the television signal in frame scan and specific maxima in the frequency area, multiple frame and linear harmonics, containing useful information, band filters should be supplemented by the basic frequencies of these maxima. The harmonics of the frame frequency carry information about the large details of the image and practically, without significant reduction in the recognition quality, the low-frequency part of the spectrum can be combined.

Experimental verification of this statement has shown that when separating from the television signal of the spectrum of low frequencies $(50-500 \mathrm{~Hz})$ and at constant time within 0.5 seconds with a period of rotation of the image 2-3 seconds, accurately, simple geometric monochrome shapes such as triangles, squares, rectangles, etc are described. Functions, received at the filter output, are close to contours. To distinguish the classes of these figures, there are sufficient simple features such as the number of transitions of the redistribution function through zero, the number of positive or negative impulses.

At frequencies, corresponding to the harmonics of linear scan, describing images of moderate complexity it is necessary to take into account the information of a larger volume - practically to the 20th harmonic. Higher frequencies provide information about small details of complex images. 


\section{RESULTS AND DISCUSSION}

Solving practical problems, there are often cases where the decision to attach an image $S$ to a certain image is based on incomplete data, that is, when not all $n$ elements of the description (attributes) can be measured.

The problem of finding an optimal decisive rule arises, which, in a number of cases, can be constructed on the basis of a method based on the definition of the maximum incomplete coefficient of similarity. In [12], [15], [24] four such methods are given.

1. Let the given image $S=S\left(x_{1}, \ldots, x_{n-k}\right)$, where $k$ - the number of missing signs. Then, to determine the belonging of this image to one of two images, use the following rule:

$$
S \in V_{1} \text { if } \frac{P\left(V_{1} / x_{1}, \ldots, x_{n-k}\right)}{P\left(V_{2} / x_{1}, \ldots, x_{n-k}\right)}>1
$$

Considering that for each image

$$
P\left(V_{1} / x_{1}, \ldots, x_{n-k}\right)=P\left(V_{1}\right) \frac{P\left(x_{1}, \ldots, x_{n-k} / V_{1}\right)}{P\left(x_{1}, \ldots, x_{n-k}\right)}
$$

the decision rule can be formulated as follows:

$$
S \in V_{1} \text { if } \frac{P\left(x_{1}, \ldots, x_{n-k} / V_{1}\right)}{P\left(x_{1}, \ldots, x_{n-k} / V_{2}\right)}>\frac{P\left(V_{2}\right)}{P\left(V_{1}\right)}
$$

The decisions, made on the basis of this rule, may vary greatly from the optimal ones.

2. Let's assume that it is possible to determine the most probable values of missing attributes. Then we can make an expression for conditional density of the probabilities of the following form:

$$
P\left(x_{1}, \ldots, x_{n-k}, x_{n-k+1}^{*}, \ldots, x_{n}^{*} / V_{1}\right) \text { and } P\left(x_{1}, \ldots, x_{n-k}, x_{n-k+1}^{*}, \ldots, x_{n}^{*} / V_{2}\right),
$$

where $x^{*}$ - probable values of signs.

The decision rule can be formulated as follows:

$$
S \in V_{1} \text { if } \frac{P\left(x_{1}, \ldots, x_{n-k}, x_{n-k+1}^{*}, x_{n}^{*} / V_{1}\right)}{P\left(x_{1}, \ldots, x_{n-k}, x_{n-k+1}^{*}, x_{n}^{*} / V_{2}\right)}>\frac{P\left(V_{2}\right)}{P\left(V_{1}\right)}=\theta
$$

3. There are conditions in which there are no $n-k$ measurements, and the similarity coefficient is a random function of non-measurable coordinates $x_{n-k+1}, \ldots, x_{n}$. In this case, the similarity coefficient can be determined by the formula:

$$
L(x)=\frac{P_{V_{1}}\left(x_{n-k+1}, \ldots, x_{n} / x_{1}, x_{2}, \ldots, x_{n-k}\right)}{P_{V_{2}}\left(x_{n-k+1}, \ldots, x_{n} / x_{1}, x_{2}, \ldots, x_{n-k}\right)}
$$

where probabilities $P_{V 1}$ and $P_{V 2}$ relate to images $V_{l}$ and $V_{2}$ accordingly.

With a similarity coefficient $L(x)$ the probability density $P[L(x)]$ is bounded. In this regard, there is such value of $L^{*}(x)$, that maximizes $P[L(x)]$ value, so $P\left[L^{*}(x)\right]=\max P[L(x)]$.

Then the decision rule can be written as follows:

$$
S \in V_{1} \text { if } L^{*}(x)>\theta
$$

4. Considering the similarity coefficient as a random function of the missing measurements, the average value of the decision rule may be used in its construction:

$$
\overline{L(x)}=\int_{-\infty}^{\infty} L(x) P[L(x)] d L
$$

In this case the decision rule can be formulated as follows:

$$
S \in V_{1} \text { if } \overline{L(x)}>\theta
$$


Comparing different decision rules, it is necessary to determine which rules give the least probability of error. For this purpose, it is necessary to calculate the probability of errors of the first and second series $P_{a}$ and $P_{b}$ In the general case, we can write it as follow:

$$
\begin{aligned}
& P_{a}=P\left(V_{2}\right) \int_{\Omega_{1}} \int \ldots \int P\left(x_{1}, \ldots, x_{n-k} / V_{2}\right) d x_{1}, \ldots, d x_{n-k}, \\
& P_{a}=P\left(V_{1}\right) \int_{\Omega_{2}} \int \ldots \int P\left(x_{1}, \ldots, x_{n-k} / V_{1}\right) d x_{1}, \ldots, d x_{n-k} .
\end{aligned}
$$

The difference between the decision rules is determined only by the choice of the area of integration. We will assume that in the area $\Omega_{1}^{c}$, the decision rule $c$ will choose an image $V$, and in the area $\Omega_{2}^{c}-$ an image $V_{2}$.

Considering the cost of the error of the first and the second kind, $\delta_{a}$ and $\delta_{b}$, then, two different decision rules can be compared, taking into account the value of risk for them. So, if $W_{c}>W_{d}$, then the decisive rule $c$ is better than $d$.

The risk or the average penalty value for this decision rule can be obtained from the formula:

$$
W_{c}=P\left(V_{1}\right) \delta_{b} \int_{\Omega} \ldots \int\left[\begin{array}{l}
P\left(V_{2}\right) \delta_{a} P\left(x_{1}, \ldots, x_{n-k} / V_{2}\right)- \\
-P\left(V_{2}\right) \delta_{b} P\left(x_{1}, \ldots, x_{n-k} / V_{1}\right)
\end{array}\right] d x_{1}, \ldots, d x_{n-k}
$$

As it was shown earlier, the minimum value $W_{c}$ is achieved with such a choice of the area $\Omega^{c}$, in which the subintegral expression would always be negative, that is,

$$
\delta_{b} P\left(V_{1}\right) P\left(x_{1}, \ldots, x_{n-k} / V_{1}\right)>\delta_{b} P\left(V_{2}\right) P\left(x_{1}, \ldots, x_{n-k} / V_{2}\right)
$$

If the cost of the error of the first and the second kind are the same, that is, if $\delta_{a}=\delta_{b}$ then area $\Omega^{c}$, in which $S \in V_{1}$ should be chosen so that

$$
\frac{P\left(x_{1}, \ldots, x_{n-k} / V_{1}\right)}{P\left(x_{1}, \ldots, x_{n-k} / V_{2}\right)}>\frac{P\left(V_{2}\right)}{P\left(V_{1}\right)}
$$

The expression (17) is a rule (8). Therefore, it can be argued that, no introduction of additional probabilistic information about unknown signs, in the case of equality of cost of errors, can give rules better than rule (6).

Before that we proceeded from the assumption, that the statistical properties of collections of educational images and images, which are encountered in recognition, remain constant. Often in the learning sequence images depicting various images that are not subject to interference are presented, and in the process of recognition, images, distorted by noise, are analyzed. In addition, if the training of the information subsystem is conducted considering of the obstacles, then the statistical properties of these obstacles may eventually be unstable. The learning process itself is limited in time. Therefore, recognizable images may differ significantly from those images that were used in the learning sequence. In this case, the optimal solution, obtained during the learning process, will no longer be optimal for the recognition process, and hence the probability of error increases.

Figure 2 shows the qualitative dependences of the probability of error from the noise level [25]-[29]. In the event that the decision rule will be optimal for each noise level, then the error probability increases with increasing noise and reaches the limit at the value of 0.5 (dotted curve). 


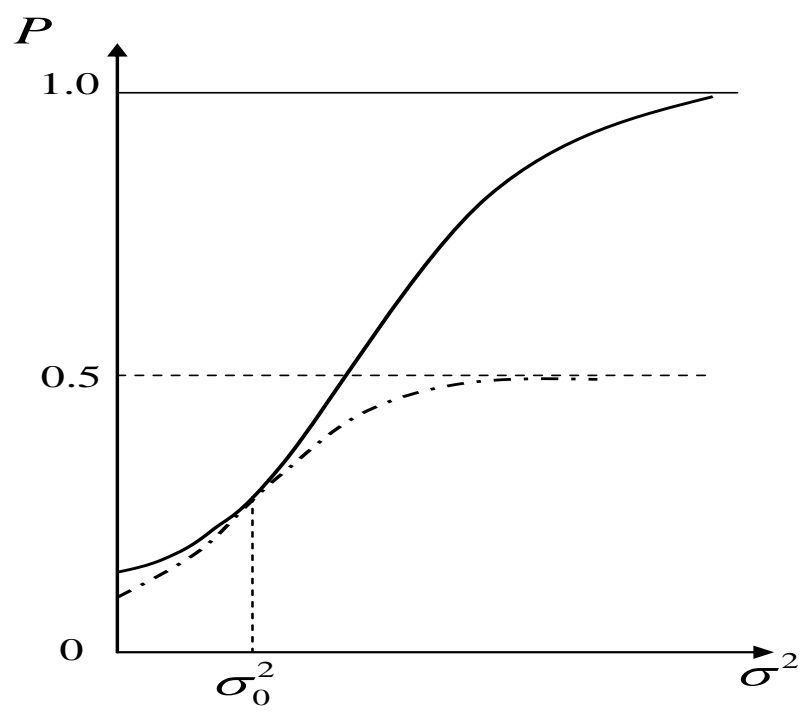

Figure 2. The error dependence on noise

If the optimal decision rule is found only for one of any value of the noise level, and in the further process of learning is not repeated, then with the increase of the noise level, the probability of error sharply increases and can reach values close to one (solid curve). At the point corresponding to the noise level, at which the training was conducted, the two curves coincide. The assessment of the noise effect on the recognition process is a very difficult task, which, in the general case, has no analytical solution. The latter can only be obtained when the educational images and audible noises are distributed according to normal law. Almost always, when solving practical problems, it is assumed that at small deviations of the statistical properties of the noise, the deviation of the probability of error from its minimum value is insignificant. In order to eliminate the effects of interference on the process of recognition, special deciding rules are introduced. One of them is based on the fact that the decision on the affiliation of the representation to the image is made on the basis of analysis of representations that have fallen into a certain closest space, which is classified. In this case, the solution takes into account the majority, which is in keeping with the results, obtained by calculating the similarity factor. Let's formulate the decision rule as follows:

$$
S \in V_{1} \text { if } \sum_{q=1}^{M_{V_{1}}}\left[1 / 1+\left(\frac{L\left(S_{1} S_{q}\right)}{r}\right)^{k}\right]>\sum_{p=1}^{M_{V_{2}}}\left[1 / 1+\left(\frac{L\left(S_{1} S_{p}\right)}{r}\right)^{k}\right]
$$

In this expression $k$ determines a plurality of images, which quite fully characterizes the whole set of images; $r$ - the radius of the area, which significantly affects the decision. The sphere with the center at the point, corresponding to the image $S$, formed by the radius $r$, will be called $r$ - region of the image $S$ is shown in Figure 3; $L\left(S, S_{q}\right)$ - measure of the similarity of the image $S$ with images belonging to the image $V_{1}$, $L\left(S, S_{p}\right)$ - a measure of similarity of the image $S$ with images belonging to the image $V_{2}$.

For discussion, consider the following. Thus, for a rather large meaning $k$, this rule defines the number of the representation of the images $V_{1}$ that are contained within a sphere with a radius $r$ centered at the point, corresponding to the image $S$. This number is compared with the number of representation $V_{2}$ of the images in the same sphere. 


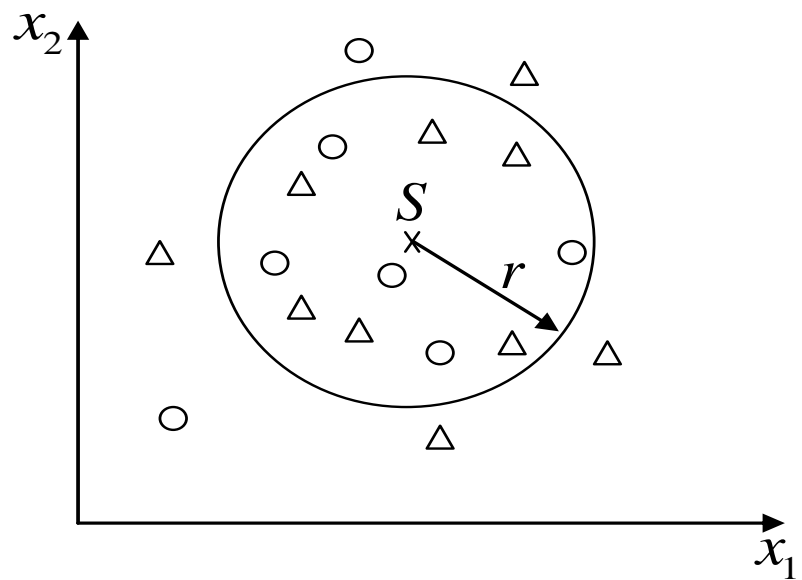

Figure 3. The information circle of the image

With this method, solutions are found that are almost unintelligible to the errors that arise due to the effects of interference. Obviously, with increasing $k$ this rule goes over to the Bayes' rule [29, 30].

\section{CONCLUSION}

In this paper, the process of identification is considered in the case of incomplete input information. The complexity of this process is to find such a description in which the image (information) of each class would have defined similar properties. The problem of finding the optimal decisive rule arises, which in some cases can be constructed on the basis of a method based on the definition of the maximum incomplete coefficient of similarity. To eliminate the influence of interference on the recognition process, special decision rules are introduced.

One of them is based on the fact that the decision on the belonging of the image to the image is made on the basis of the analysis of the images that fall into a certain close space, is classified. In this case, the decision takes into account the majority, which agrees well with the results obtained by calculating the similarity coefficient. This allows you to find solutions that are almost not sensitive to errors that occur due to interference.

\section{REFERENCES}

[1] Zhang K, Wang X. Motion fuzzy images reduction of high-voltage line inspection based on spectrum analysis and image autocorrelation. Proceedings of the 2017 IEEE International Conference on Robotics and Biomimetics (ROBIO), China, 2017; 1160 - 1164.

[2] Attamimi M, Mardiyanto R, Irfansyah AN. Inclined Image Recognition for Aerial Mapping using Deep Learning and Tree based Models. TELKOMNIKA Telecommunication, Computing, Electronics and Control. 2018; 16(6): 3034-3044.

[3] Ravazzi C, Coluccia G, Magli E. Curl-Constrained Gradient Estimation for Image Recovery From Highly Incomplete Spectral Data. IEEE Transactions on Image Processing. 2017; 26(6): 2656 - 2668.

[4] Sheugh L, Alizadeh SH. A note on pearson correlation coefficient as a metric of similarity in recommender system. Proceedings of the 2015 AI \& Robotics (IRANOPEN), Iran, 2015; 1-6.

[5] Zhang L, Mahapatra D, Tielbeek Jeroen AW, and other. Image Registration Based on Autocorrelation of Local Structure. IEEE Transactions on Medical Imaging. 2016; 1(35): 63-75.

[6] Zhu C, Yu L, Yan Z, Xiang S. Frequency Estimation of the Plenoptic Function Using the Autocorrelation Theorem. IEEE Transactions on Computational Imaging. 2017; 3(4): 966-981.

[7] Humennyi D, Parkhomey I, Tkach M. Structural model of robot-manipulator for the capture of non-cooperative client spacecraft. Advances in Intelligent Systems and Computing. 2018; 754: 33-42.

[8] Hazra T, CRS K, Nene MJ. Strategies for Searching Targets Using Mobile Sensors in Defense Scenarios. International Journal of Intelligent Systems and Applications. 2017; 9(5): 61-70.

[9] Arifin AS, Wahyuni DK, Suryanegara M, Asvial M. Ship Speed Estimation using Wireless Sensor Networks: Three and Five Sensors Formulation. TELKOMNIKA Telecommunication, Computing, Electronics and Control. 2018; 16(4): 1527-1534.

[10] Lu J. Recursive fourier-based high-frame rate imaging. Proceedings of the 2014 IEEE International Ultrasonics Symposium, USA, 2014; 121-124. 
[11] Nathan KS, Beigi HSM, Subrahmonia J, Clary GJ, Maruyama H. Real-time on-line unconstrained handwriting recognition using statistical methods. Proceedings of the 1995 International Conference on Acoustics, Speech, and Signal Processing, Detroit, 1995; 2619-2622.

[12] Tang D, Yang Y, Fu S. Semi-bent functions with perfect three-level additive autocorrelation. Proceedings of the 2017 Eighth International Workshop on Signal Design and Its Applications in Communications (IWSDA), Sapporo, 2017; 164-168.

[13] Jaafar H, Ismail NS. Intelligent Person Recognition System Based on ECG Signal. Telecommunication, Electronic and Computer Engineering. 2018: 10(1-13): 83-88.

[14] Akshaya R, Hema P Menon. A Review on Registration of Medical Images Using Graph Theoretic Approaches. Indonesian Journal of Electrical Engineering and Computer Science. 2018; 12(3): 974-983.

[15] Sheugh L, Alizadeh SH. A note on pearson correlation coefficient as a metric of similarity in recommender system. Proceedings of the 2015 AI \& Robotics (IRANOPEN), Qazvin, 2015; 1-6.

[16] Sun J, Lv Q, Tan Z, Liu Y. An image sharpening strategy based on multiframe super resolution for multispectral data. Proceedings of the 2016 8th Workshop on Hyperspectral Image and Signal Processing: Evolution in Remote Sensing (WHISPERS), USA, 2016; 1-5.

[17] Leow CH, Braga M, Stanziola A, and other. Multi-frame rate plane wave contrast-enhance ultrasound imaging for tumour vasculature imaging and perfusion quantification. Proceedings of the 2017 IEEE International Ultrasonics Symposium (IUS), USA, 2017; 1-4.

[18] Erwin E, Saparudin S, Saputri W. Hybrid Multilevel Thresholding and Improved Harmony Search Algorithm for Segmentation. International Journal of Electrical and Computer Engineering. 2018; 8(6): 4593-4602.

[19] Wen X, Qiao L, Ma S, and other. Sparse Subspace Clustering for Incomplete Images. Proceedings of the 2015 IEEE International Conference on Computer Vision Workshop (ICCVW), Chile, 2015; 859-867.

[20] Arun SK, Ravi K. An Efficient Filtering Technique for Denoising Colour Images. International Journal of Electrical and Computer Engineering. 2018; 8(5): 3604-3608.

[21] Sankaran S, Sethumadhavan G. Entropy-Based Colour Splitting in Dermoscopy Images to Identify Internal Borders. Proceedings of the 2018 International Conference on Inventive Research in Computing Applications (ICIRCA), Coimbatore, 2018; 771-774.

[22] Chiara R, Sophie F, Tiziano B, Enrico M. Sparsity estimation from compressive projections via sparse random matrices. EURASIP Journal on Advances in Signal Processing. 2018; 2018(56).

[23] Oktiana M, Fitri A, Away Y, Munadi K. Features for Cross Spectral Image Matching: A Survey. Bulletin of Electrical Engineering and Informatics. 2018; 7(4): 552-560.

[24] Reddy A, Mungara J. Wireless Environment Aware Adaptive Scheduling Technique For Cellular Networks. Indonesian Journal of Electrical Engineering and Computer Science. 2018; 11(1): 318-332.

[25] Boiko J, Eromenko O. Signal Processing in Telecommunications with Forward Correction of Errors. Indonesian Journal of Electrical Engineering and Computer Science. 2018; 11(3): 868-877.

[26] Huang HC, Chen PL, Chang FC. Error Resilient Transmission for Compressed Sensing of Color Images with Multiple Description Coding. Proceedings of the 2015 Third International Conference on Robot, Vision and Signal Processing (RVSP), Kaohsiung, 2015; 63-66.

[27] Parhomey IR, Boiko JM, Eromenko OI. Features of digital signal processing in the information control systems of multipositional radar. Journal of Achievements in Materials and Manufacturing Engineering. 2016; 2(77): 75-84.

[28] Shynkaruk O, Boiko J, Eromenko O. Measurements of the energy gain in the modified circuit signal processing unit. Proceedings of the 2016 13th International IEEE Conference on Modern Problems of Radio Engineering. Telecommunications and Computer Science (TCSET), Ukraine, 2016; 582-584.

[29] Dewi YN, Riana D, Mantoro T. Improving Nä̈ve Bayes performance in single image pap smear using weighted principal component analysis (WPCA). Proceedings of the 2017 International Conference on Computing, Engineering, and Design (ICCED), Malaysia, 2017; 1-5.

[30] Jahromi AH, Taheri M. A non-parametric mixture of Gaussian naive Bayes classifiers based on local independent features. Proceedings of the 2017 Artificial Intelligence and Signal Processing Conference (AISP), Shiraz, 2017; 209-212. 